\title{
Residual Stress Field and Reduction of Stress Intensity Factors in Coldworked Holes
}

\author{
S.T. Pinho*, H.B. Martins*, P.P. Camanho ${ }^{\dagger}$, \\ M.H. Santare ${ }^{\ddagger}$ and P.M.S.T. de Castro*
}

\begin{abstract}
Analytical closed-form and semi-analytical solutions for the residual stress distributions in a plate caused by pressure acting on a central circular hole, representing the cold-work process, are derived for an elastic-perfectly plastic material. Both Tresca and von Mises yield criteria are used and the corresponding residual stress distributions are compared. The relation between the dimension of the plastic zone and the value of internal pressure is presented. The relation between the magnitude of the residual stresses and the remote uniform tensile stress required to open symmetrical radial cracks is also presented. The reduction of the stress intensity factors of cracked open and riveted holes as a function of the internal pressure applied (or mandrel radial displacement) is investigated using numerical models for both an elastic-perfectly plastic material and for an Al 2024-T3 Alclad aluminum alloy.
\end{abstract}

\section{Notation}

$\Delta \bar{r}$ Non-dimensional radial increment

$\Delta \ldots \ldots$. Hole radial expansion (difference between mandrel and hole radius)

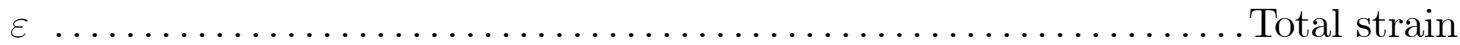
$\varepsilon_{i j}^{p l} \ldots \ldots \ldots \ldots \ldots \ldots \ldots \ldots \ldots \ldots \ldots \ldots \ldots \ldots \ldots \ldots \ldots \ldots$ component of the plastic strain

\footnotetext{
*Faculdade de Engenharia, Universidade do Porto- Structural Integrity Unit, IDMEC, Rua Roberto Frias, 4200-465-Porto, Portugal

${ }^{\dagger}$ Faculdade de Engenharia, Universidade do Porto- Structural Integrity Unit, IDMEC, Rua Roberto Frias, 4200-465-Porto, Portugal- pcamanho@fe.up.pt- Corresponding Author

${ }^{\ddagger}$ Department of Mechanical Engineering, University of Delaware, Newark, Delaware 19716, U.S.A.
} 


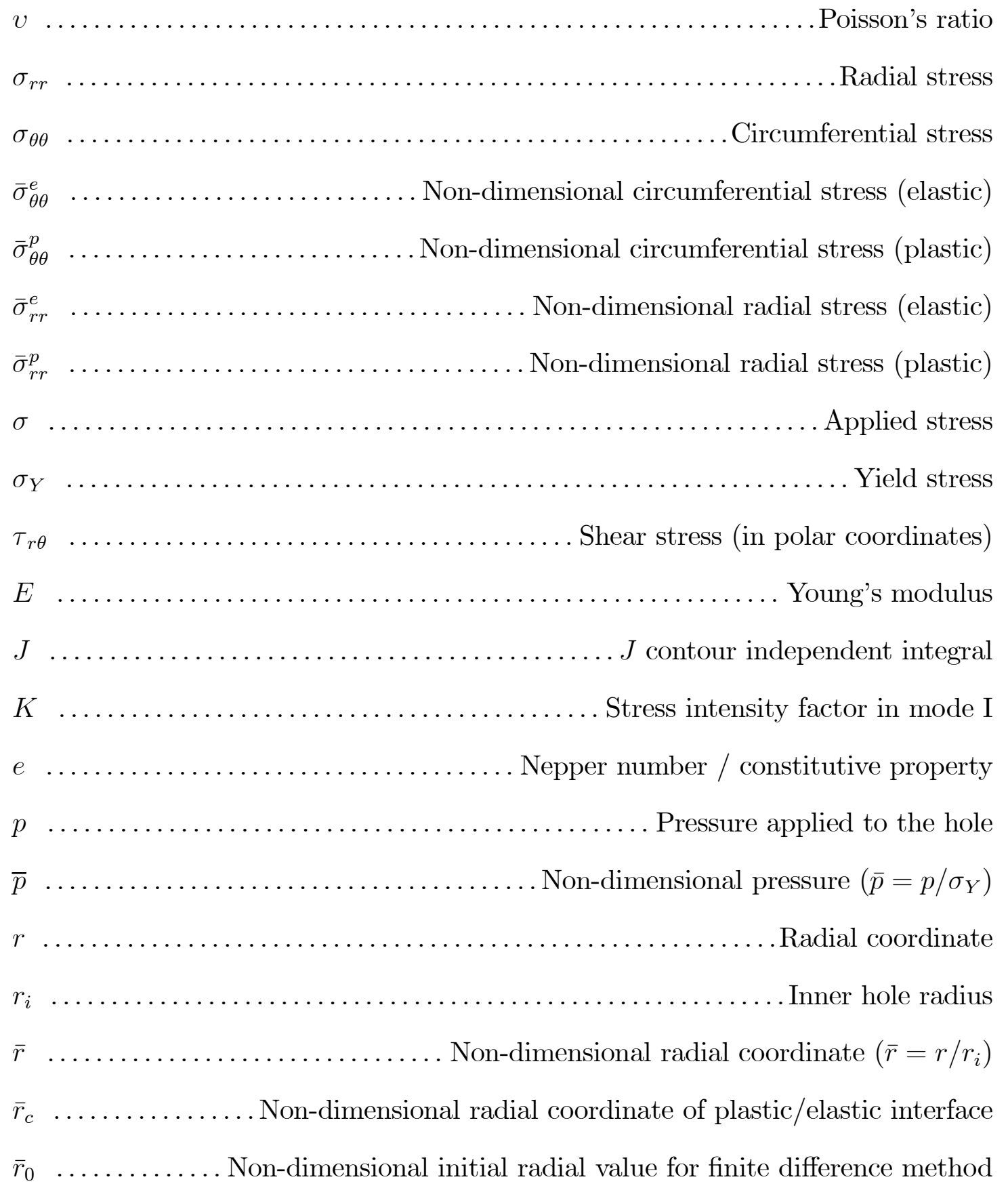




\section{Introduction}

The number of problems related with ageing aircraft may be reduced by enhancing the fatigue performance of aeronautical structures, especially in critical zones, acting as stress raisers, such as access and riveted holes.

Fastener hole fatigue resistance may be enhanced by creating compressive residual circumferential stresses around the hole. This technique - cold-work - has been used in the aeronautical industry for the past thirty years to delay fatigue damage and retard crack propagation.

Research has been concentrated mainly on modelling the residual stress field using analytical or two-dimensional (2D) or three-dimensional (3D) numerical methods $[1,5]$, on the experimental measurement of the residual stress field $[6,7]$, on the experimental characterization of the cold-worked hole behaviour in fatigue $[8,10]$, and on the stress intensity factor calibration for cracks that may nevertheless develop after cold-work $[2,11,12]$. Subtopics considered include the consideration of thickness effects [5-13], the consideration of eventual pre-existence of cracks of various sizes before hole expansion is carried out [8], the possible re-cold-working of already cold-worked holes [14], and the stress analysis of neighbouring cold-worked holes [15].

The compressive circumferential residual stress field around the rivet holes is created by applying pressure on the hole surface by means of a mandrel. The applied pressure must be large enough to plasticize the material surrounding the hole. Once the pressure is removed, the desired residual compressive stress field is achieved. According to Leon [16], the main benefits associated with the improvement of the fatigue life are the reduction of unscheduled maintenance, increasing the time between inspection intervals, reduction of maintenance costs, and improvement of aircraft readiness.

Two cold-working processes are normally used in the aeronautical industry [16, 17]: the split sleeve process, using a solid tapered mandrel and a lubricated split sleeve, and the split mandrel process, using a lubricated, hollow, and longitudinally slotted tapered mandrel.

In service conditions, cracks may initiate and grow from the surface of the hole. However, due to the compressive residual stress, there will be a minimum value of remote tensile stress required to open the crack. Furthermore, once the cracks are open, the respective stress intensity factor, $K$, will be smaller than the one obtained in the absence of cold-working. Therefore, the cold-working process retards crack growth, increasing the fatigue life of the structure.

This process is shown on Figure 1. The reduction of $K$ depends on the magnitude of the residual stresses, on the size and location of the $\operatorname{crack}(\mathrm{s})$, on the edge effects introduced by the particular geometry, and on the material used.

Since the reduction of the stress intensity factor is a function of the residual 
stresses, it is important to relate the magnitude of the residual stress field with the expansion of the mandrel (or with the pressure applied to the rivet hole) when designing a riveted connection. The relation between the residual stress field and the pressure applied to the hole can be obtained by means of Finite Element Analyses (FEA), or using experimental stress analysis techniques (e.g. strain gages, photoelastic coatings, laser interferometry). However, FEA analyses require preand post-processing, trained users, and are time consuming. Experimental stress analyses are expensive, time consuming and clearly impractical for the analysis of different hole sizes, materials and internal pressures.

The objectives of the current work are to develop a simplified procedure to relate the internal pressure applied to the hole of an infinite isotropic plate with the residual stress field and plastic zone, and to assess the reduction of stress intensity factors resulting from the cold-work process in both open and riveted holes.

The availability of a simplified procedure to relate internal pressure with plastic zones is important in the design of a riveted connection, avoiding more complex, expensive and time-consuming analyses. The analytical and semi-analytical procedures, proposed to deal with stages a) and b) represented in Figure 1, are compared with finite element solutions.

After the characterization of the residual stress field, the effects of cold-working on the stress intensity factor of an elastic-perfectly plastic material, and of an aluminium alloy plate containing symmetrically located cracks, are assessed (stages c) and d) represented in Figure 1). The effects of cold-work on the stress intensity factor are investigated for both an open hole remotely loaded and for a riveted joint.

\section{Closed-form analytical and semi-analytical so- lutions for residual stress fields}

The residual stress field obtained by the cold-work process is derived using the Tresca and von Mises yield criteria, assuming an elastic-perfectly plastic material.

\subsection{Residual stress field using Tresca criterion}

It is considered that the cold-work process creates a symmetric pressure distribution around the hole. This assumption, used in majority of the numerical models proposed, holds true for the split mandrel cold-working process, but is not sufficiently accurate for split sleeve cold-working [16]. It is also considered that the hole is expanded by a rigid mandrel, and that the material does not contain residual stresses due to manufacturing. 
The elastic stresses due to an internal pressure, $p$, on a circular hole with a radius $r_{i}$, in an infinite plate can be easily derived from Lamé's equations [18], considering plane stress state and neglecting frictional effects:

$$
\begin{aligned}
\sigma_{r r}(r) & =-p\left(\frac{r_{i}}{r}\right)^{2} \\
\sigma_{\theta \theta}(r) & =p\left(\frac{r_{i}}{r}\right)^{2} \\
\tau_{r \theta} & =\sigma_{z z}=0
\end{aligned}
$$

The Tresca criterion can be expressed by:

$$
\sigma_{1}-\sigma_{3}=\sigma_{Y}
$$

where $\sigma_{Y}$, the yield stress, is considered constant (elastic-perfectly plastic behaviour assumed).

For the case of axisymmetric pressure, equations (1)-(3), this can be reduced to:

$$
\sigma_{1}-\sigma_{3}=\sigma_{\theta \theta}-\sigma_{r r}=2 p\left(\frac{r_{i}}{r}\right)^{2}=\sigma_{Y}
$$

Therefore, the internal pressure that leads to yielding onset, $p_{o}$, occurring for $r=r_{i}$, is obtained as:

$$
p_{o}=\frac{\sigma_{Y}}{2}
$$

The equilibrium equation in the radial direction can be obtained as:

$$
\frac{d \sigma_{r r}}{d r}+\frac{1}{r}\left(\sigma_{r r}-\sigma_{\theta \theta}\right)=0
$$

For $p \geq p_{o}$, equation (4) is satisfied. Using (7) and (4) the equilibrium equation in the radial direction of the plastic zone is:

$$
\frac{d \sigma_{r r}}{d r}+\frac{1}{r} \sigma_{Y}=0
$$

Integrating (8), taking into account that $\sigma_{r r}\left(r_{i}\right)=-p$ :

$$
\sigma_{r r}^{p}=\sigma_{Y} \ln \frac{r}{r_{i}}-p
$$

From (4):

$$
\sigma_{\theta \theta}^{p}=\sigma_{Y}\left(\ln \frac{r}{r_{i}}+1\right)-p
$$


Equations (9) and (10) are valid for the plastic region, $r_{i} \leq r \leq r_{c}$, where $r_{c}$ delimits the elastic and the plastic zones. The stress state for the elastic region of the plate can be obtained using Lamé's equations applied to an hole with a radius $r_{c}$ and subjected to an internal pressure $p=\sigma_{r r}^{p}\left(r_{c}\right)$. Therefore, the radial and tangential stresses are:

$$
\begin{aligned}
\sigma_{r r}^{e} & =\left(\sigma_{Y} \ln \frac{r_{c}}{r_{i}}-p\right)\left(\frac{r_{c}}{r}\right)^{2} \\
\sigma_{\theta \theta}^{e} & =-\left(\sigma_{Y} \ln \frac{r_{c}}{r_{i}}-p\right)\left(\frac{r_{c}}{r}\right)^{2}
\end{aligned}
$$

The radius corresponding to the boundary between the elastic and the plastic zones, $r_{c}$, can be obtained from:

$$
\sigma_{\theta \theta}^{e}\left(r_{c}\right)=\sigma_{\theta \theta}^{p}\left(r_{c}\right) \therefore r_{c}=r_{i} e^{\left(p / \sigma_{Y}-1 / 2\right)}
$$

Defining the following nondimensional parameters:

$$
\begin{aligned}
\bar{\sigma}_{r r} & =\frac{\sigma_{r r}}{\sigma_{Y}} \\
\bar{\sigma}_{\theta \theta} & =\frac{\sigma_{\theta \theta}}{\sigma_{Y}} \\
\bar{r} & =\frac{r}{r_{i}} \\
\bar{r}_{c} & =\frac{r_{c}}{r_{i}} \\
\bar{p} & =\frac{p}{\sigma_{Y}}
\end{aligned}
$$

the stress field in the plastic zone, $1 \leq \bar{r} \leq \bar{r}_{c}$, may be defined as:

$$
\begin{aligned}
& \bar{\sigma}_{r r}^{p}=\ln \bar{r}-\bar{p} \\
& \bar{\sigma}_{\theta \theta}^{p}=\ln \bar{r}+1-\bar{p}
\end{aligned}
$$

and in the elastic zone, $\bar{r}>\bar{r}_{c}$, as:

$$
\begin{aligned}
\bar{\sigma}_{r r}^{e} & =(\ln \bar{r}-\bar{p})\left(\frac{\bar{r}_{c}}{\bar{r}}\right)^{2} \\
\bar{\sigma}_{\theta \theta}^{p} & =-\bar{\sigma}_{r r}^{e}
\end{aligned}
$$


As long as the unloading is purely elastic, the residual stress field may be obtained by the superposition principle. Therefore, the residual stress field is obtained for the plastic zone $1 \leq \bar{r} \leq \bar{r}_{c}$, as:

$$
\begin{aligned}
& \bar{\sigma}_{r r}^{p(r e s)}=\ln \bar{r}-\bar{p}\left(1-\frac{1}{\bar{r}^{2}}\right) \\
& \bar{\sigma}_{\theta \theta}^{p(r e s)}=\ln \bar{r}+1-\bar{p}\left(1+\frac{1}{\bar{r}^{2}}\right)
\end{aligned}
$$

and in the elastic zone, $\bar{r}>\bar{r}_{c}$, as:

$$
\begin{aligned}
\bar{\sigma}_{r r}^{e(r e s)} & =\left(\ln \bar{r}_{c}-\bar{p}\right)\left(\frac{\bar{r}_{c}}{\bar{r}}\right)^{2}+\frac{\bar{p}}{\bar{r}^{2}} \\
\bar{\sigma}_{\theta \theta}^{e(r e s)} & =-\bar{\sigma}_{r r}^{e(r e s)}
\end{aligned}
$$

For the Tresca yield criterion, the superposition principle is valid for a range of applied pressure smaller than twice the pressure required to initiate plastic deformation, $1 / 2 \leq \bar{p} \leq 1$ : when $\bar{p} \leq 1 / 2$ there is no plastic deformation, whereas for $\bar{p}>1$ there is plastic deformation on the hole boundary during unloading.

\subsection{Residual stress field using von Mises criterion}

The von Mises criterion can be expressed by:

$$
\frac{1}{2}\left[\left(\sigma_{1}-\sigma_{2}\right)^{2}+\left(\sigma_{2}-\sigma_{3}\right)^{2}+\left(\sigma_{3}-\sigma_{1}\right)^{2}\right]=\sigma_{Y}^{2}
$$

From (1)-(3):

$$
\frac{1}{2}\left[\left(\sigma_{r r}-\sigma_{\theta \theta}\right)^{2}+\sigma_{\theta \theta}^{2}+\sigma_{r r}^{2}\right]=\sigma_{Y}^{2}
$$

From (1), (2) and (28) the internal pressure corresponding to yielding onset is obtained as:

$$
p_{o}=\frac{\sigma_{Y}}{\sqrt{3}}
$$

Using von Mises yield criterion, the analytical integration of the governing equations for the elastic-plastic stress field is not possible. Therefore, the problem is analytically reduced to an ordinary differential equation and solved by finite differences. The results based upon this algorithm will be referred to as semianalytical. The finite-differences procedure shown in Table 1 is easily implemented in a spreadsheet. 


\subsection{Comparison between analytical/semi analytical and nu- merical solutions}

The analytical and semi-analytical solutions proposed are compared with a finite element solution. The finite element model created provides an approximate framework against which to assess the analytical and semi-analytical solutions proposed here.

ABAQUS [20] software is used to simulate one-quarter of an $800 \mathrm{~mm} \times 800 \mathrm{~mm}$ plate containing a $10 \mathrm{~mm}$ diameter hole. An elastic-perfeclty plastic material with $E=71400 \mathrm{MPa}, v=0.3$ and $\sigma_{Y}=285 \mathrm{MPa}$ is used. The resulting model has 2305 8-node, reduced integration shell elements.

In order to validate the finite element model, a simulation of a linear-elastic model is performed. The stress concentration factor predicted is 3.001, which is in good agreement with the theoretical solution (3.000).

The residual stresses obtained by the semi-analytical solution, and by the finite element model using von Mises criterion are shown in Figure 2.

From Figure 2 it can be concluded that an excellent agreement between the semi-analytical and the numerical solution is obtained. The comparison between the results predicted by the analytical and semi-analytical solutions using Tresca and von Mises criteria respectively is presented in Figure 3.

Figure 3 shows that significant differences in the residual circumferential stresses are obtained using of Tresca or von Mises yield criteria. Since most metals, including aluminum, are better represented by von Mises yield criterion, only this criterion will be used in the following sections. The numerical model also provides the relation between the radial displacement and the applied pressure. This displacement corresponds to the radial interpenetration, i.e., the difference between the mandrel and the hole radius. Figure 4 shows the relationship between the mandrel interpenetration and the pressure applied to the hole obtained by the finite element model.

\subsection{Remote stress required to open a crack}

A plate with a central circular hole, with a circumferential residual stress field (Figure 2), and subjected to an uniform remote tensile stress is considered.

In an elastic process, the superposition principle is applicable and the resulting stress field is shown in Figure 5.

Considering the presence of two symmetrically located cracks in the compressive region near the hole, the cracks will not open unless their length is greater than the radial dimension of the region under compressive stress.

This dimension is defined by the residual stress magnitude and by the remote load magnitude (see Figure 5). Therefore, it is possible to represent the remote 
uniform tensile stress required to open the cracks as a function of the crack length and residual stress magnitude by using the proposed semi-analytical procedure. This relationship is shown in Figure 6.

\section{Effect of cold-work on stress intensity factors}

After proposing a methodology to determine the relation between the plastic zone and the internal pressure applied to the hole, it is important to relate the stress intensity factors and the plastic zone. The relation between the stress intensity factors and the size of the plastic zone, for both elastic perfectly plastic material and Al 2024 Alclad aluminum alloy, is obtained using ABAQUS Finite Element software [20]. Two situations are investigated: a plate with an open hole remotely loaded, and a plate with a riveted joint.

\subsection{Open hole}

The geometry investigated consists of an infinite plate with a central circular hole. For symmetry reasons, the numerical model represents a quarter of the plate, with 2305 elements of the type S8R (8-noded, reduced integration plane stress elements) and a total of 7120 nodes. The hole diameter considered is $20 \mathrm{~mm}$ and the plate dimensions are $800 \mathrm{~mm} \times 800 \mathrm{~mm}$. Two symmetrically located $6 \mathrm{~mm}$ long cracks are simulated by releasing the boundary conditions of the corresponding nodes. Before determining the effect of cold-work on the stress intensity factors it is necessary to validate the numerical algorithm used. The accuracy of the algorithm is assessed by comparing the predicted stress intensity factor with the analytical solution described in [21]:

$$
K=Y \sigma \sqrt{\pi a}
$$

For the geometry used, $Y=1.0571$ [21]. Considering an uniform remote stress of $100 \mathrm{MPa}$, the stress intensity factor is $K=23.700 \mathrm{MPa} \sqrt{\mathrm{m}}$.

Values of the $J$-integral [22] are calculated for twenty different contour paths around the crack tip. Convergence is obtained after the third path and small oscillations are observed as the $J$-integral contours approach the surface of the hole. The stress intensity factor can be obtained from the $J$-integral value, considering plane stress, as:

$$
K=\sqrt{J E}
$$

Using equation (31) the predicted value of the stress intensity factor is $K=23.714 \mathrm{MPa} \sqrt{\mathrm{m}}$, corresponding to a difference of $0.06 \%$ when compared with the analytical solution [21]. 
Two different materials are investigated: an elastic perfectly plastic material with $E=71400 \mathrm{MPa}, v=0.3$ and $\sigma_{Y}=285 \mathrm{MPa}$, and $\mathrm{Al} 2024$ Alclad aluminum alloy with $E=71400 \mathrm{MPa}, v=0.3$ and $\sigma_{Y}=285 \mathrm{MPa}$. The uniaxial stress-strain relation for $\mathrm{Al} 2024$ Alclad is required to define the elasto-plastic behaviour the material. This relation can be obtained in [23], and is given by:

$$
\varepsilon=\left\{\begin{array}{c}
\sigma / E, \sigma<\sigma_{Y} \\
\left(\sigma_{Y} / E\right)\left(\sigma / \sigma_{Y}\right)^{n}, \sigma \geq \sigma_{Y}
\end{array}\right.
$$

where $n=8$ for $\mathrm{Al} 2024$ Alclad. Associated plastic flow, with isotropic yielding is assumed.

Three load cases corresponding to different values of internal pressures $(65 \%$, $75 \%$ and $85 \%$ of the yield stress) are investigated. Associated with these internal pressures, the corresponding hole radial expansions, $\Delta$, and the non-dimensional radius corresponding to the plastic zone, $\bar{r}_{c}$, are represented in Table 2 .

A first analysis is performed to obtain the residual stress field. An uniform remote tensile stress is then applied, completely opening the crack. The $J$-integral calculations in the presence of residual stresses presented some convergence problems for contours in the vicinity of the hole surface. Nevertheless, the stable region of the curve allows the calculation of stress intensity factors.

The influence of the different values of residual stresses on $K$ is obtained for several crack lengths and is shown in Figure 7 for the two materials investigated.

Figure 7 shows that cold-work reduces the stress intensity factor for the two materials investigated. The reduction of the stress intensity factor is more pronounced in the elastic-perfectly plastic material, for an internal pressure corresponding to $85 \%$ of yield stress, and for the shortest crack length used, $a=12.5 \mathrm{~mm}$. Under this circumstances, the reduction of the stress intensity factor when compared with a hole without cold-work is $25.4 \%$. It is also worth noticing that for a crack length $a=12.5 \mathrm{~mm}$ and for an internal pressure corresponding to $85 \%$ of yield stress, the crack lies in a region totally plastically deformed by the cold-work process, as shown in Figure 8. This fact can explain the significant reduction of the stress intensity factor obtained for both materials for a short crack length and high internal pressure during the cold-work.

\subsection{Riveted hole}

The effect of cold-work is investigated for a double-shear riveted joint, where there is no bending of the plate.

The rivet is simulated as a rigid body and a frictionless contact between the rivet and the plate is assumed. The contact problem is addressed using a variational formulation constrained by the contact conditions using Lagrange multipliers [20]. The contact surface and the distribution of radial stresses are not assumed, 
but calculated throughout the analysis using the contact algorithm in ABAQUS [20]. The same materials used in the plate with an open hole, an elastic perfectly plastic material and an $\mathrm{Al} 2024$ Alclad aluminum alloy, are investigated.

Half of an $200 \mathrm{~mm} \times 200 \mathrm{~mm}$ plate containing a $20 \mathrm{~mm}$ diameter hole is simulated using 8-node plane stress elements and using appropriated boundary conditions along the symmetry plane. A total of 3762 elements and 11396 nodes are used in the numerical model. Before simulating the presence of a plastic zone due to the cold-working process, the accuracy of the algorithm is assessed by comparing the predicted stress intensity factor with the solution obtained by Cartwright and Parker [24]. Considering 5 different crack lengths, values of the $J$-integral [22] are calculated for 10 different paths, and the converged values are used to calculate the stress intensity factor using (31). The stress intersity factors normalized using $K_{0}=\sigma \sqrt{\pi a}$ obtained by the numerical models are compared with the values obtained in [24], and the results are shown in Figure 9.

For the crack lengths used, differences on the stress intensity factors between 6.4 and $8.1 \%$ are obtained. These differences can be justified by the fact that the methodology proposed in [24] uses a cosine function to simulate the distribution of radial stresses, whereas the numerical model implemented here calculates the radial stresses using a contact algorithm. The difference between the cosine and calculated distributions of radial contact stress is shown in Figure 10.

Three load cases corresponding to different values of internal pressures (65\%, $75 \%$ and $85 \%$ of the yield stress) are investigated. The corresponding hole radial expansions, $\Delta$, and the non-dimensional radius corresponding to the plastic zone, $\bar{r}_{c}$, are represented in Table 3.

The simulation of a riveted joint with cold-worked holes is performed in three steps. The first step simulates the cold-work process, where an internal pressure (or radial displacement) is applied to the hole. Internal pressures corresponding to $65 \%, 75 \%$ and $85 \%$ of the yield stress are considered. In the second step the internal pressure is removed, allowing the elastic recovery of the material. In the third step the contact conditions between a $20 \mathrm{~mm}$ diameter rivet and the hole are defined, two symmetrically located cracks are simulated, and the $J$-integral is calculated for ten different paths.

The stress intensity factor, $K$, is represented in Figure 11 for the different cases investigated as a function of the crack length.

Figure 11 shows that cold-work reduces the stress intensity factor for the two materials investigated. Like in the plate with an open hole, the highest reduction of the stress intensity factor occurs for the elastic-perfectly plastic material, for an internal pressure corresponding to $85 \%$ of the yield stress, i.e., the higher hole radial expansion considered in the present work. For this case, the highest reduction of the stress intensity factor is $4.3 \%$, when compared with a hole without cold-work. For internal pressures smaller than $85 \%$ of the yield stress there is almost no 
difference between the behaviour of the two materials, and marginal reductions of the stress intensity factor are obtained using the cold-work process. Like in the case of a plate with an open hole, for a crack length $a=12.5 \mathrm{~mm}$ and for an internal pressure corresponding to $85 \%$ of yield stress, the crack lies in a region totally plastically deformed by the cold-work process.

\section{Conclusions}

Analytical and semi-analytical solutions are derived for the characterization of the residual stress field and plastic zone sizes of cold-worked holes for elastic-perfectly plastic materials. The analytical and semi-analytical solutions can predict the residual stress field, and relate the cold-working pressure to both the dimension of plastically deformed region and to the dimension of the region subjected to compressive stresses. The proposed solutions are also able to relate the remote uniform tensile stress required to open a crack, for several values of crack lengths, and to the dimension of the plastic zone. The solutions do not require complex pre- or post-processing techniques, being therefore useful tools for the design of cold-worked holes.

The cold-working process is shown to reduce stress intensity factors for different dimensions of plastically deformed regions, and for different crack lengths, for both a remotely loaded plate containing an open hole, and for a riveted joint. The maximum reductions of the stress intensity factor obtained are $25.4 \%$ and $4.3 \%$ for open and riveted holes, respectively. The maximum reduction for the open hole case occurs in the situation where the all the length of the crack lies in the plastically deformed region created by cold-work.

The reduction of stress intensity factor value for the cracked open hole problem is greater than the one obtained for the pin-loaded joint. This implies that claims of fatigue enhancement solely based on the performance of open hole specimens may be optimistic in actual structural connections.

The constitutive law used affects the reduction of the stress intensity factor due to cold-work: higher reductions of the stress intensity factor are obtained in elastic-perfectly plastic materials.

Even for the small values of radial expansion considered in the present work, fatigue strength enhancing consequences are predicted, namely through the need for a minimum remote tensile stress in order to open cracks of a given size, the reduction of $K$ for the cracked open hole problem, and the reduction, although small, of $K$, for the pin loaded joint models analysed. 


\section{Ackowledgements}

The present work is part of the contribution of IDMEC to the ADMIRE project (project GRD1-2000-25069; contract G4RD-CT-2000-0396) of the Commission of the European Community. Prof Santare's visit to the University of Porto was made possible through a grant from the Fulbright Commission. 


\section{References}

[1] J. Kang, W. S. Johnson, D. A. Clark, 'Three-dimensional finite element analysis of the cold expansion of fastener holes in two aluminium alloys', Journal of Engineering Materials and Technology, Transactions of the ASME, vol. 124, 140-145 (2002).

[2] D. L .Ball, 'Elastic-plastic stress analysis of cold expanded fastener holes', Fatigue and Fracture of Engineering Materials and Structures 18, (1), 47-63 (1995).

[3] C. Poussard, M. J. Pavier, D. J. Smith, 'Analytical and finite element predictions of residual stresses in cold-worked fastener holes', Journal of Strain Analysis 30, (4), 291-304 (1995).

[4] G. Wanlin, 'Elastic-plastic analysis of a finite sheet with a cold-worked hole', Engineering Fracture Mechanics 45, (6), 857-864 (1993).

[5] G. Clark, 'Modelling residual stresses and fatigue crack growth at coldexpanded fastener holes', Fatigue and Fracture of Engineering Materials and Structures 14, (5), 579-589 (1991).

[6] G. A. Webster, A. N. Ezeilo, 'Residual stress distribution and their influence on fatigue lifetimes', International Journal of Fatigue 23, S375-S383, (2001).

[7] M. Priest, C. G. Poussard, M. J. Pavier, D. J. Smith, 'An assessment of residual-stress measurements around cold-worked holes', Experimental Mechanics, 361-366 (1995).

[8] D. L. Ball, D. R. Lowry, 'Experimental investigation on the effects of cold expansion of fastener holes', Fatigue and Fracture of Engineering Materials and Structures 21, 17-34 (1998).

[9] O. Buxbaum, H. Huth, 'Expansion of cracked fastener holes as a measure for extension of lifetime to repair', Engineering Fracture Mechanics 28, (5/6), 689-698 (1987).

[10] L. Schwarmann, 'On improving the fatigue performance of a double-shear lap joint', International Journal of Fatigue 5, (2),105-111 (1983).

[11] A. F. Grandt Jr, 'Stress intensity factors for some through-cracked fastener holes', International Journal of Fracture 11, (2),.283-294 (1975). 
[12] A. F. Grandt Jr, J. P. Gallagher, 'Proposed fracture mechanics criteria to select mechanical fasteners for long service lives', in: 'Fracture Toughness and Slow-stable Cracking', ASTM STP 559, 283-297 (1974).

[13] R. A. Pell, P. W. Beaver, J. Y. Mann, J. G. Sparrow, 'Fatigue of thicksection cold-expanded holes with and without cracks', Fatigue and Fracture of Engineering Materials and Structures, 12, (6), 553-567 (1989).

[14] M. Bernard, T. Bui-Quoc, M. Burlat, 'Effect of re-cold-working on fatigue life enhancement of a fastener hole', Fatigue and Fracture of Engineering Materials and Structures 18, (7/8), 765-775 (1995)

[15] P. Papanikos, 'Mechanics of mixed mode fatigue behaviour of cold worked adjacent holes', PhD Thesis, University of Toronto, (1997).

[16] A. Leon, 'Benefits of split mandrel cold-working', International Journal of Fatigue 20 (1), 1-8, (1998).

[17] J. Schijve, 'Fatigue of Structures and Materials', (Kluwer Academic Publishers, 2001).

[18] G. Lamé, 'Leçons sur la Théorie de l'Élasticité', Gauthier-Villars, Paris, (1852) (in French).

[19] A Boresi, R Schmidt, O Sidebottom, 'Advanced Mechanics of Materials', 5th edition, John Wiley \& Sons, 1993

[20] ABAQUS; User's manual - version 6.1., Hibbit, Karlsson \& Sorensen Inc., (2000).

[21] Y. Murakami, 'Stress Intensity Factors Handbook' Vol I, (Pergamon, 1987).

[22] J. R. Rice, 'A path independent integral and the approximate analysis of strain concentration by notches and cracks', Journal of Applied Mechanics 31, 379-386 (1968).

[23] T. Siegmund, W. Brocks, 'Modeling crack growth in thin sheet aluminium alloys', Fatigue and Fracture Mechanics: 31st Volume, ASTM STP 1389, G R Halford and J P Gallagher, Eds., American Society for Testing and Materials, 475-485 (2000).

[24] D. J. Cartwright, A. P. Parker, 'Opening mode stress intensity factors for cracks in pin-loaded joints', International Journal of Fracture 18 (1), 65-78 (1982). 


\section{Tables}

Table 1: Proposed finite differences procedure

\begin{tabular}{ll}
\hline \hline Condition & Expression \\
\hline Initiation & $\bar{r}_{0}=1$ (initial point for finite differences method) \\
& $\Delta \bar{r}=0.01$ (radial increment) \\
& $\left(\bar{\sigma}_{r r}\right)_{1}=-\bar{p}$ (boundary condition) \\
if $\left(\bar{\sigma}_{r r}\right)_{1}>\frac{1}{\sqrt{3}}$ & $\left(\bar{\sigma}_{\theta \theta}\right)_{1}=+\sqrt{1-\frac{3}{4}\left[\left(\bar{\sigma}_{r r}\right)_{1}\right]^{2}}+\frac{1}{2}\left(\bar{\sigma}_{r r}\right)_{1}$ (equilibrium/yield criterion) \\
else & $\left(\bar{\sigma}_{\theta \theta}\right)_{1}=-\left(\bar{\sigma}_{r r}\right)_{1}($ equilibrium/compatibility) \\
\hline Iteration $i$ & $\left(\bar{\sigma}_{r r}\right)_{i}=\left(\bar{\sigma}_{r r}\right)_{i-1}-\frac{1}{\bar{r}_{i}}\left[\left(\bar{\sigma}_{r r}\right)_{i-1}-\left(\bar{\sigma}_{\theta \theta}\right)_{i-1}\right] \times \Delta \bar{r}$ (equilibrium) \\
if $\left(\bar{\sigma}_{r r}\right)_{i}>\frac{1}{\sqrt{3}}$ & $\left(\bar{\sigma}_{\theta \theta}\right)_{i}=+\sqrt{1-\frac{3}{4}\left[\left(\bar{\sigma}_{r r}\right)_{i}\right]^{2}}+\frac{1}{2}\left(\bar{\sigma}_{r r}\right)_{i}($ equilibrium/yield criterion) \\
else & $\left(\bar{\sigma}_{\theta \theta}\right)_{i}=-\left(\bar{\sigma}_{r r}\right)_{i}($ equilibrium/compatibility) \\
\hline \hline
\end{tabular}

Table 2: Load cases for plate with open hole

\begin{tabular}{cccc}
\hline \hline & \multicolumn{3}{c}{ Elastic-perfectly plastic/Al 2024-T3 Alclad } \\
\hline Load Case & $\mathbf{1}$ & $\mathbf{2}$ & $\mathbf{3}$ \\
\hline $\bar{p}$ & 0.65 & 0.75 & 0.85 \\
\hline $\bar{r}_{c}$ & $1.065 / 1.010$ & $1.165 / 1.150$ & $1.265 / 1.250$ \\
\hline$\Delta / r_{i} \times 10^{3}$ & $3.24 / 3.42$ & $4.17 / 4.11$ & $5.19 / 5.00$ \\
\hline \hline
\end{tabular}

Table 3: Load cases for plate with rivet joint

\begin{tabular}{|c|c|c|c|}
\hline & \multicolumn{3}{|c|}{ Elastic-perfectly plastic/Al 2024-T3 Alclad } \\
\hline Load Case & 1 & 2 & 3 \\
\hline $\bar{p}$ & 0.65 & 0.75 & 0.85 \\
\hline $\bar{r}_{c}$ & $1.050 / 1.050$ & $1.150 / 1.150$ & $1.275 / 1.275$ \\
\hline$\Delta / r_{i} \times 10^{3}$ & $3.48 / 3.47$ & $4.25 / 4.19$ & $5.32 / 5.11$ \\
\hline
\end{tabular}




\section{Figure Captions}

Figure 1: Schematic representation of the cold-work process and load case studied

Figure 2: Semi-analytical and numerical residual stress fields using von Mises criterion

Figure 3: Relationship between applied pressure and radial interpenetration

Figure 4: Residual stresses obtained using von Mises and Tresca yield criteria

Figure 5: Stress field due to cold-work and remote loading

Figure 6: Stress required to open two symetrically located cracks in a coldworked hole

Figure 7: Stress intensity factor as a function of crack length and cold-working

Figure 8: Regions plastically deformed for different internal pressures

Figure 9: Comparison between stress intensity factors for a plate with a riveted hole

Figure 10: Cosine and calculated distributions of radial contact stress

Figure 11: Normalised stress intensity factor as a function of crack length and cold-work 


\section{Figures}

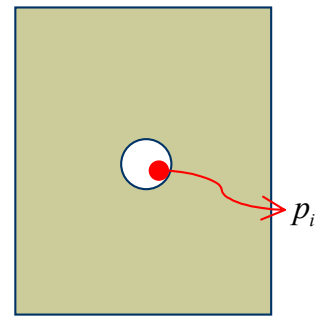

a) Infinite plate with pressure applied along central circular hole.

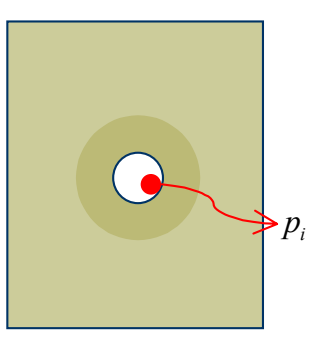

b) Region plastically deformed.

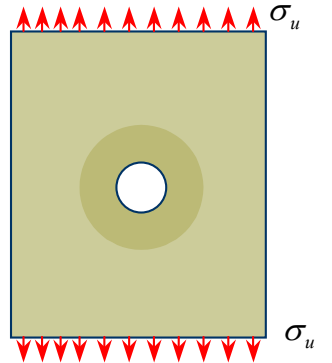

c) Uniform remote tensile stress and compressive residual stress field around the hole.

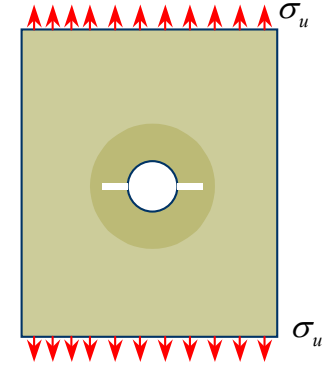

d) Circular hole with two symmetric cracks.

Figure 1: Schematic representation of the cold-work process and load case studied 


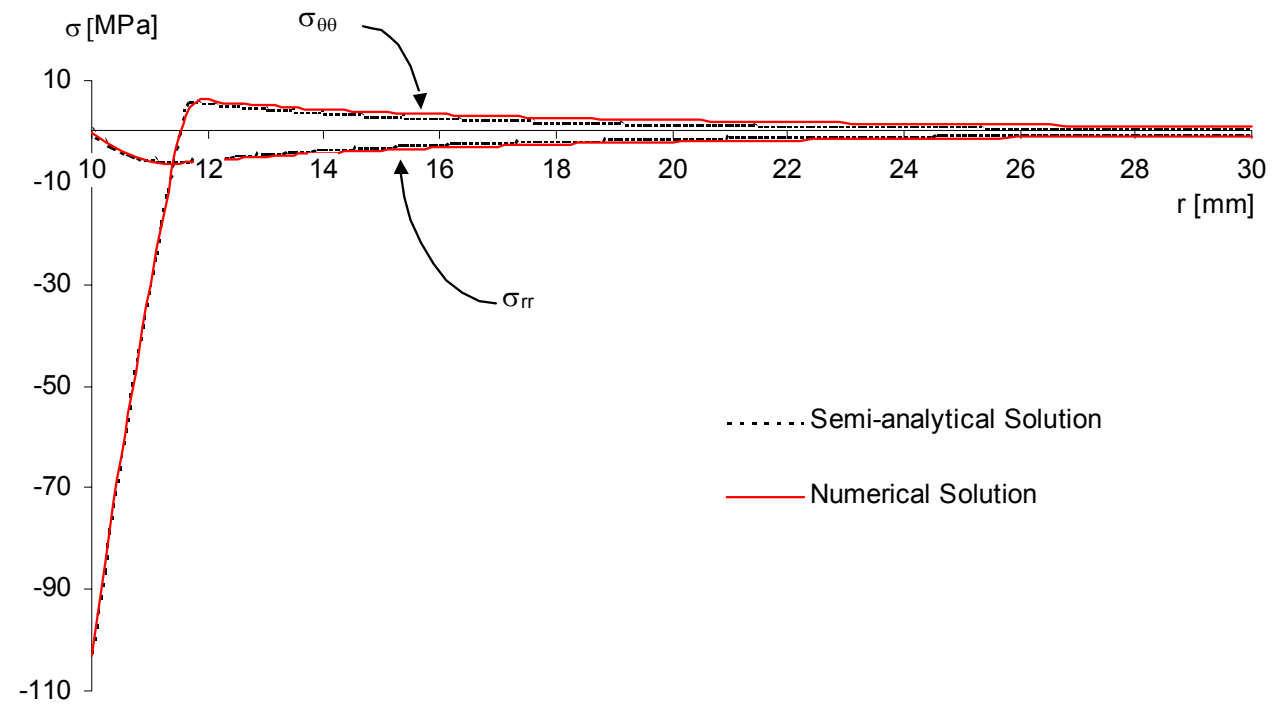

Figure 2: Semi-analytical and numerical residual stress fields using von Mises criterion

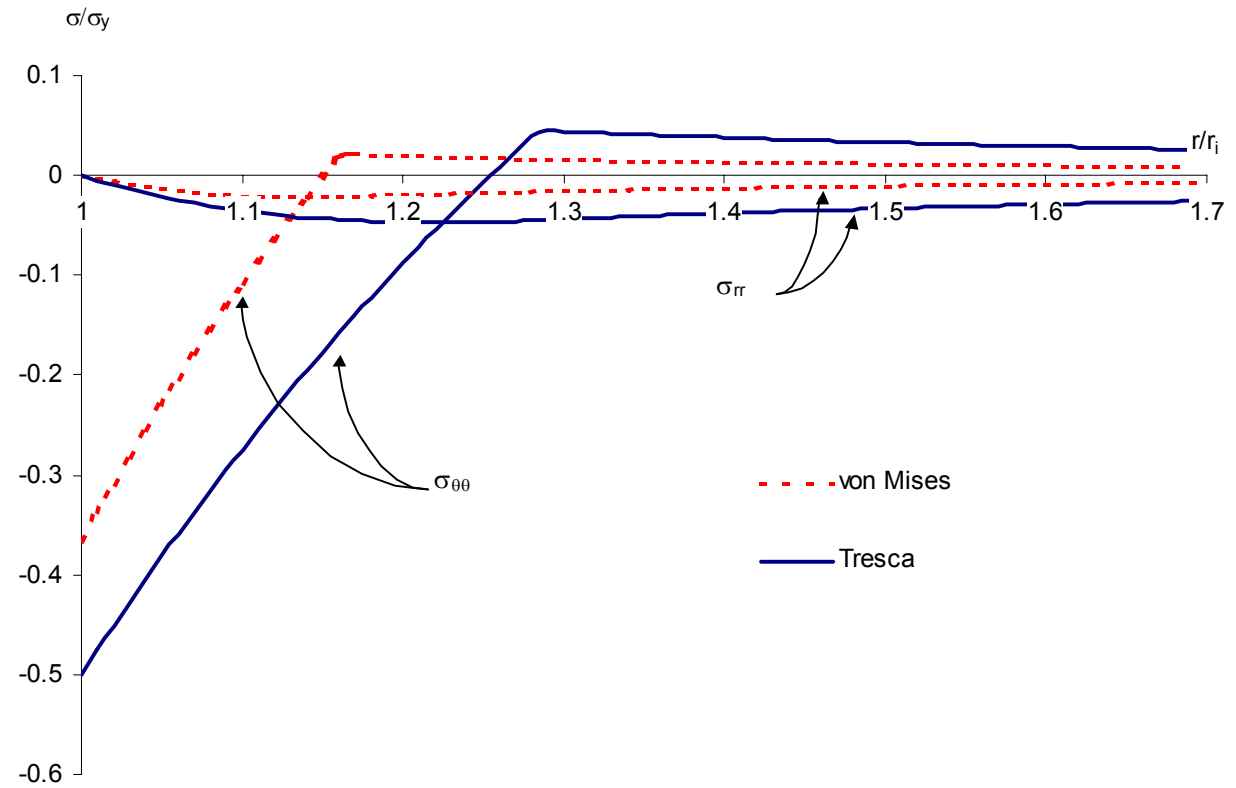

Figure 3: Residual stresses obtained using von Mises and Tresca yield criteria 


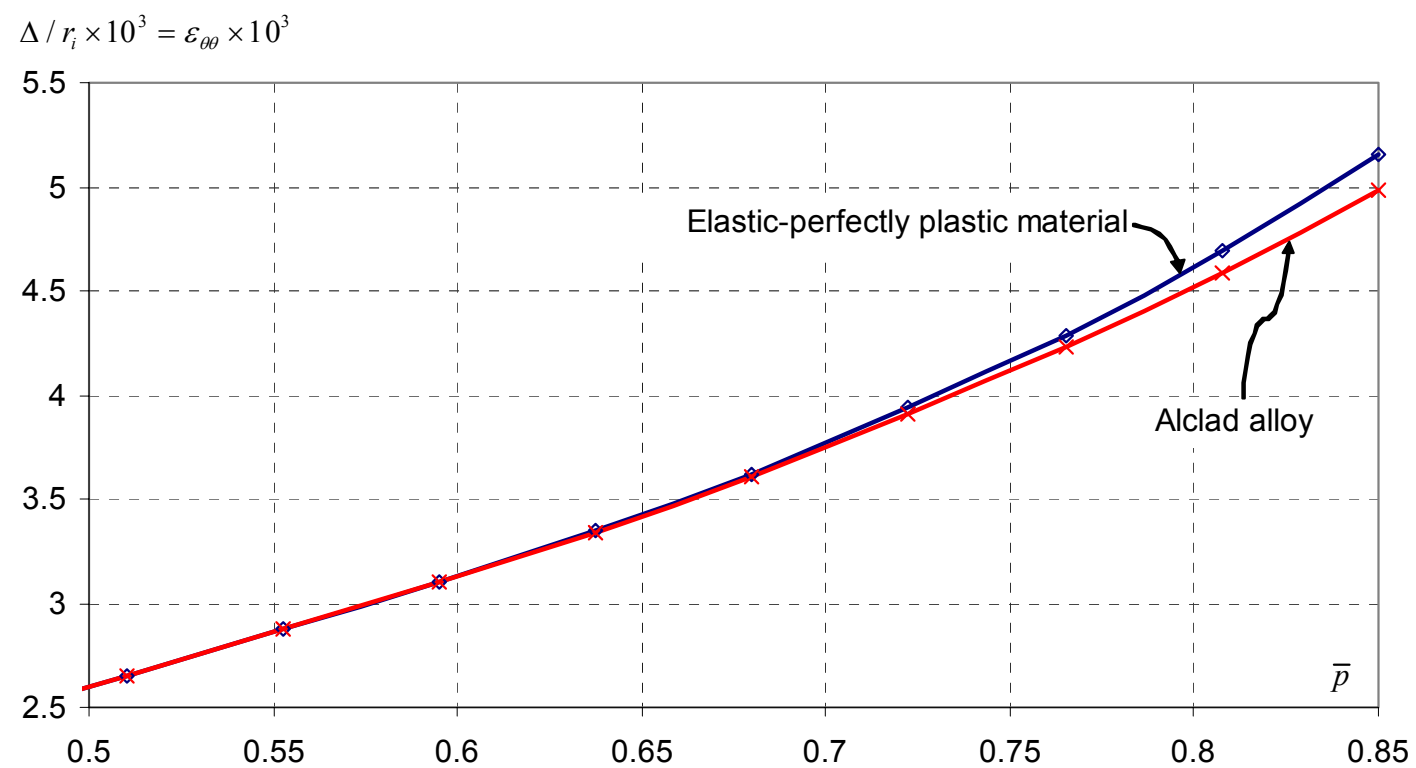

Figure 4: Relationship between applied pressure and radial interpenetration

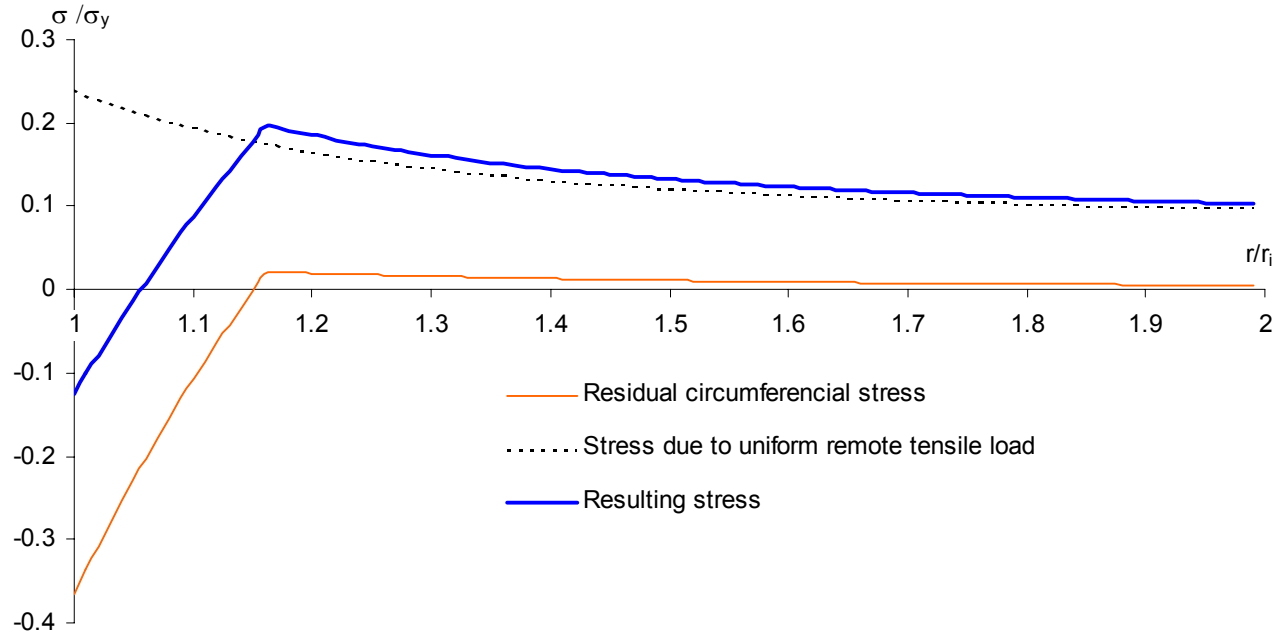

Figure 5: Stress field due to cold-work and remote loading 


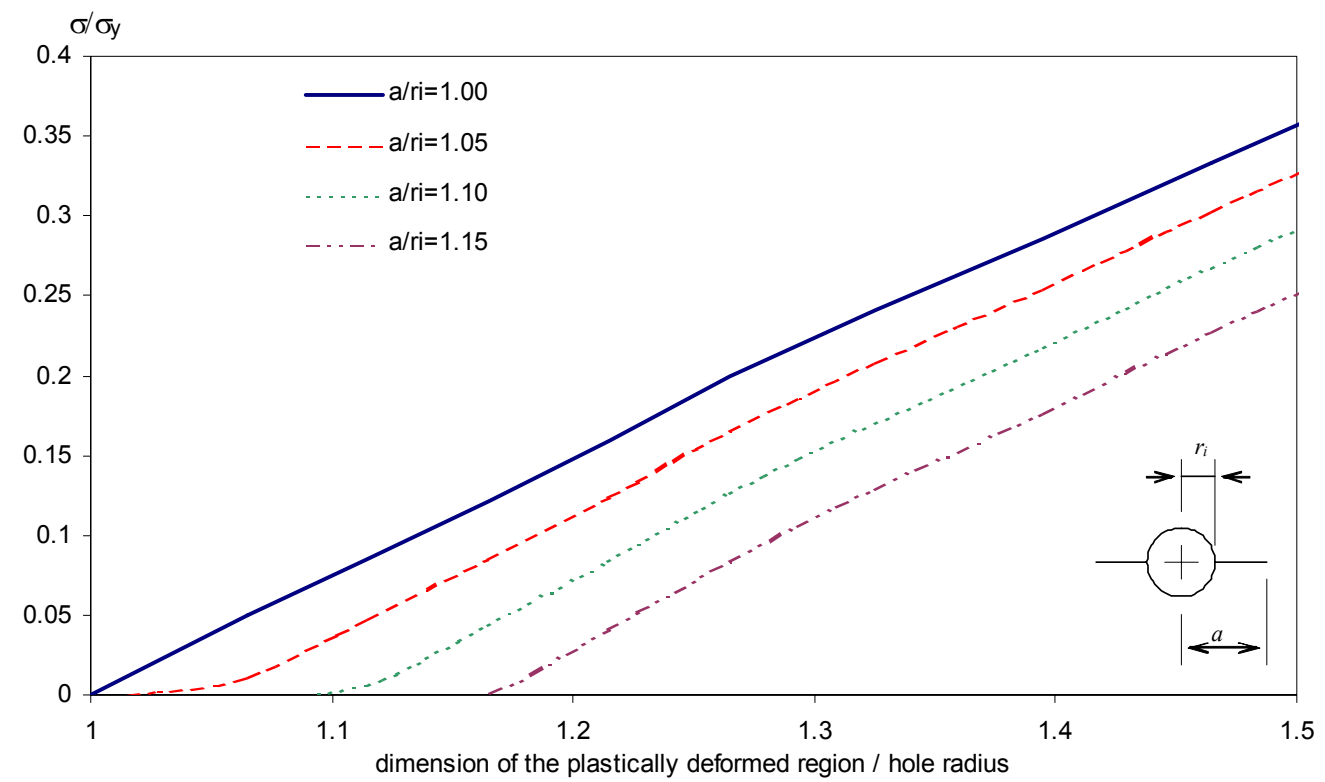

Figure 6: Stress required to open two symetrically located cracks in a cold-worked hole

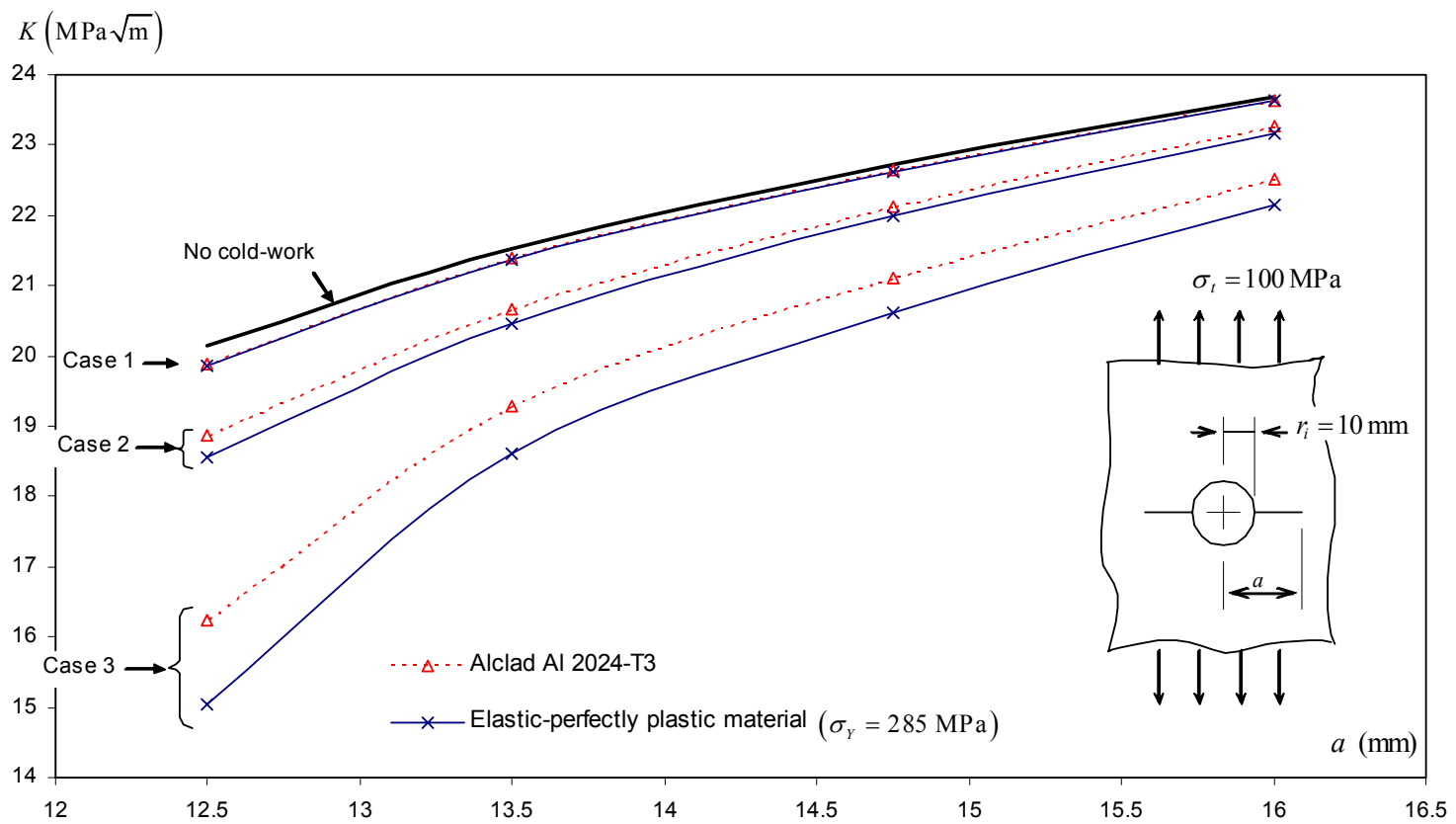

Figure 7: Stress intensity factor as a function of crack length and cold-working 


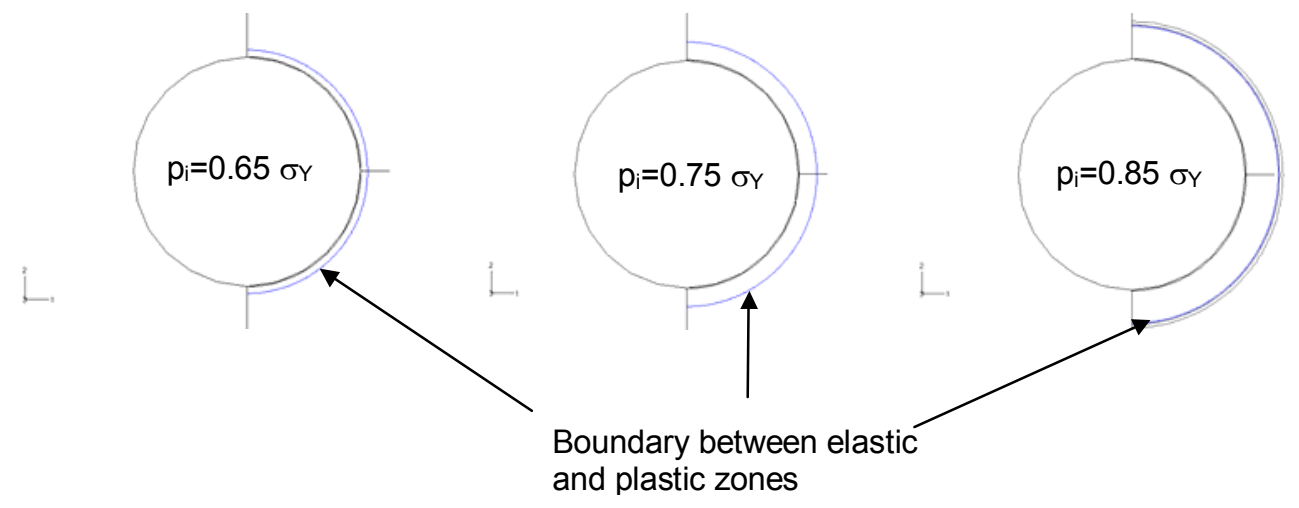

Figure 8: Regions plastically deformed for different internal pressures

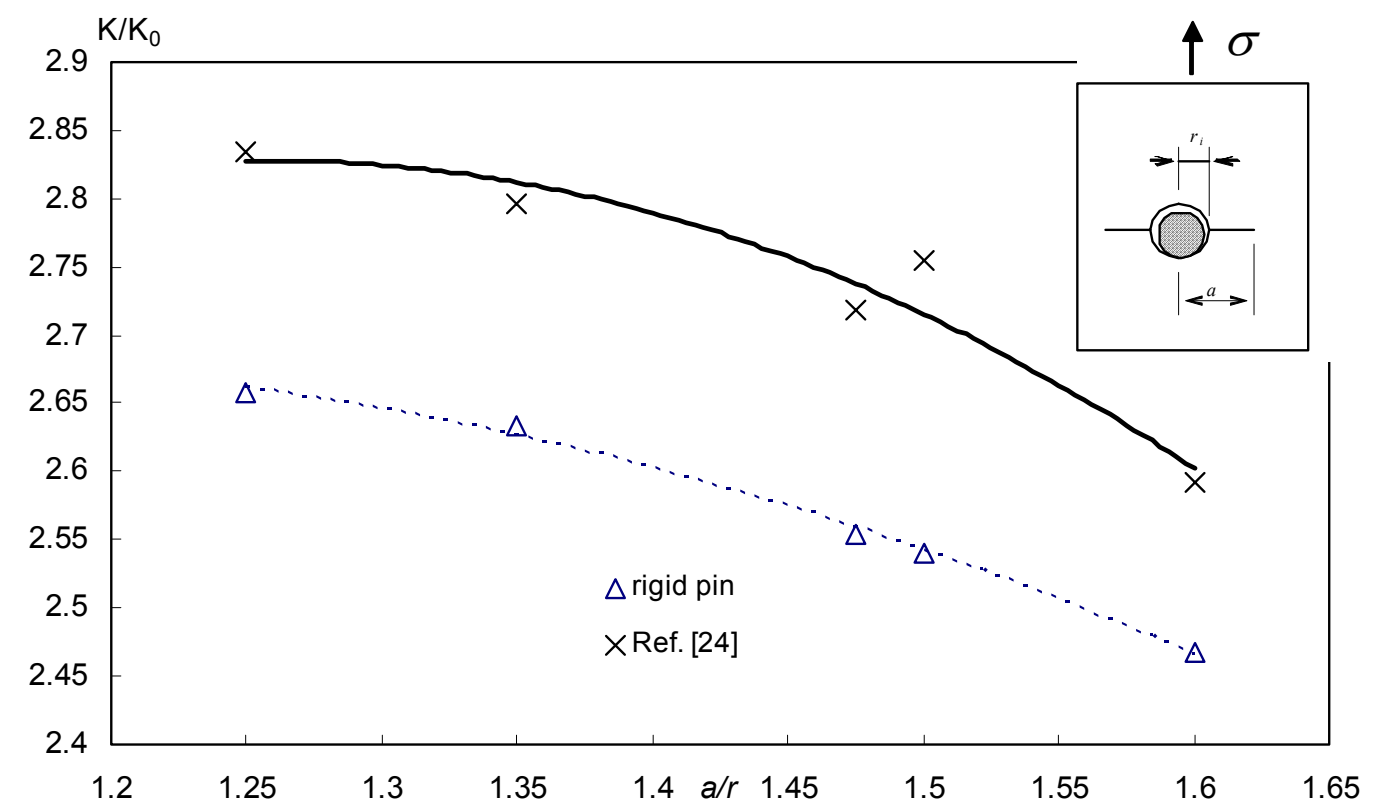

Figure 9: Comparison between stress intensity factors for a plate with a riveted hole 


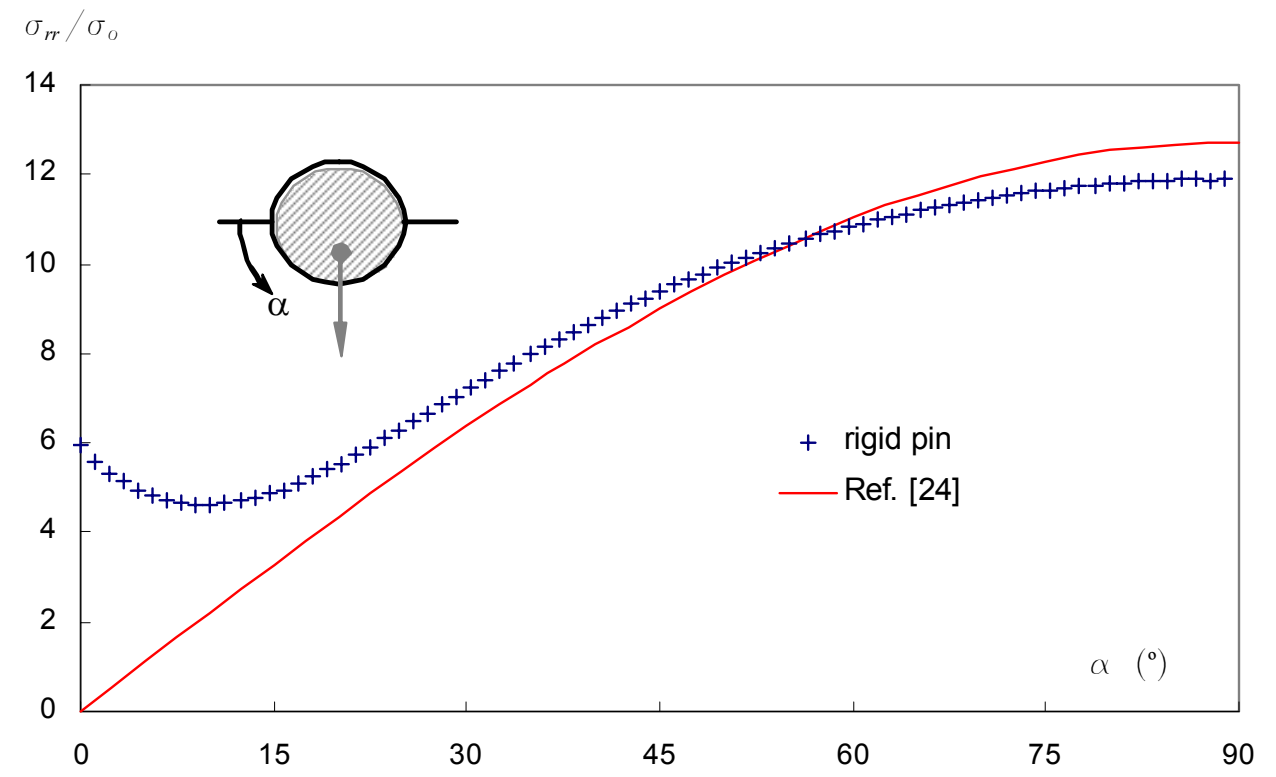

Figure 10: Cosine and calculated distributions of radial contact stress

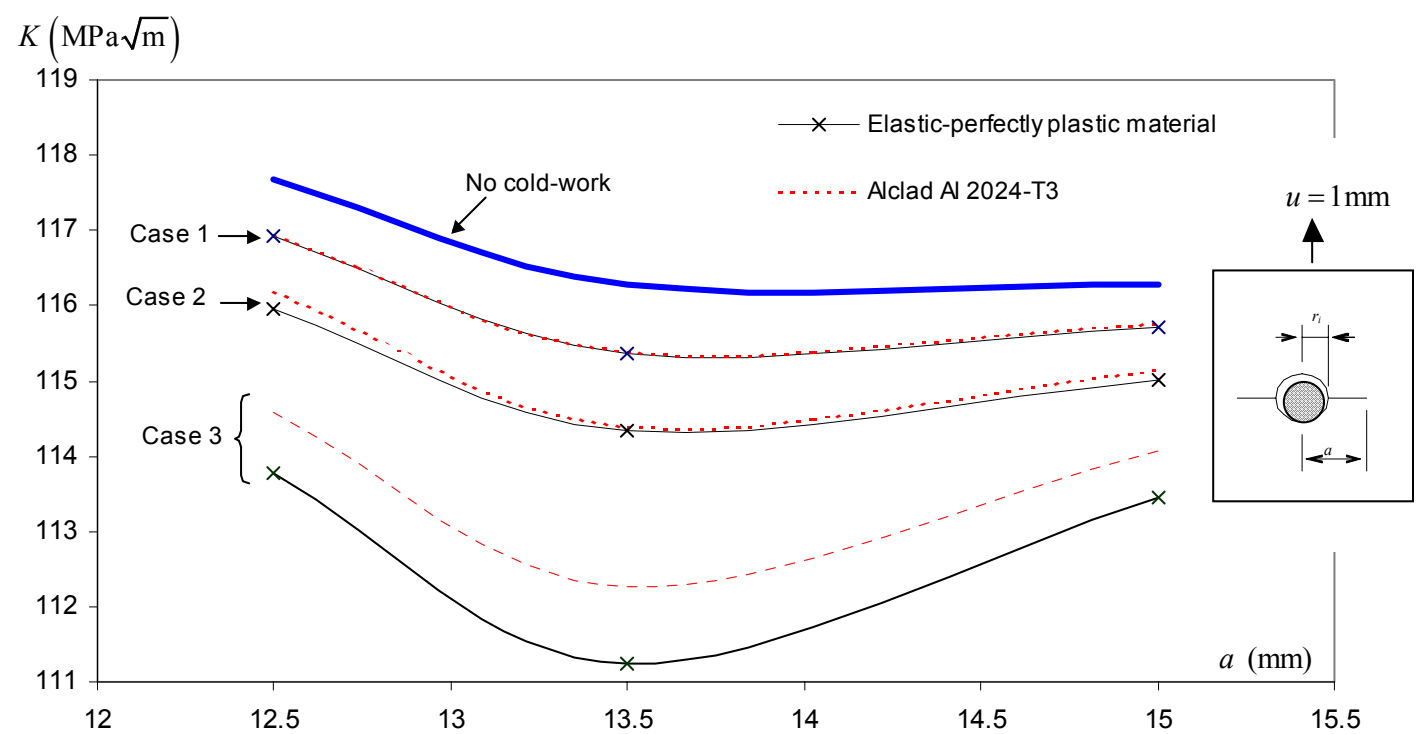

Figure 11: Normalised stress intensity factor as a function of crack length and cold-work 